\title{
EXPLORING CUBE AFFORDANCE: TOWARDS A CLASSIFICATION OF NON-VERBAL DYNAMICS OF PHYSICAL INTERFACES FOR WEARABLE COMPUTING
}

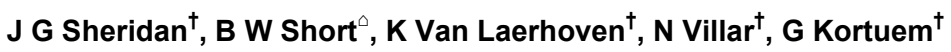 \\ ${ }^{\dagger}$ Department of Computing, Lancaster University, LA1 4YR Lancaster, United Kingdom \\ Department of Psychology, Lancaster University, LA1 4YR Lancaster, United Kingdom
}

\begin{abstract}
Current input technologies for wearable computers are difficult to use and learn and can be unreliable. Physical interfaces offer an alternative to traditional input methods. In this paper we propose that developing a well-designed physical interface requires an exploration of the psychological idea of affordance. We present our findings from a design study in which we explore the natural affordance of a cube and suggest possible requirements for the design of graspable cubeshaped physical interfaces as alternative rich-action input device. We expect that such a framework will enhance the precision and usability of devices for wearable and mobile computing.
\end{abstract}

\section{INTRODUCTION}

Using a mouse as an input device for wearable computing is problematic (1). Rich-action input devices such as ToolStone (2) and tilting interfaces (3) such as Rock'n'Scroll (4) offer alternatives to the mouse and allow effective use of human's manipulation skills.

Developing a usable and desirable (5) physical interface takes an understanding of how users build an intimate relationship with an object or how they dissociated themselves from the object they are controlling (6). In order to establish what these user desires are, we need to gain as much information as possible about the way users want to interact with objects.

Hands are the most expressive parts of the human body and are used for grasp and gesture. While gesture has played a major role in defining physical interfaces $(7,8)$, the focus of this paper is on how users physically manipulate an object, rather than how they use body and limb motions as a means of expression. Therefore, we will focus our attention on grasp rather than gesture.

We distinguish our work from the graspable user interfaces introduced by Fitzmaurice et al in that grasp here does not imply concurrence between spacemultiplexed input and output (9) but simply as a clasping or seizing motion, especially with the fingers or hands. While Buttolo provides an extensive survey of human grasping techniques (10), very little research has been dedicated to understanding how humans interact with tools $(11,12)$.

Although the physical form of an interface fundamentally shapes the kinds of interactions that users can perform (5), how users want to interact with an object is just as important as the kinds of interactions an object affords. Affordance is a psychological idea that states that objects may suggest, by their shape and other attributes, what you can do to them $(13,14)$. A rich-action input device adds some complexity in that it physically changes the way users hold the device (2).
Using the shape of a cube as an example, we explore the concept of affordance by conducting a design study. Our study aims to answer the following questions:

- How do physical properties (such as size, shape, and form) affect affordance?

- What types of manipulations are possible with cubes of various designs?

- What kinds of non-verbal dynamics does a cube afford?

We expect that natural sets of non-verbal dynamics will arise when people interact with cubes of various design specifications.

Rather than delivering an application-specific solution for the design of physical interfaces, our set of dynamics introduces a robust, scalable framework for the development of physical interfaces in general.

\section{DESIGN STUDY}

Since the purpose of this study was to understand how people naturally interact with a cube, we conducted a design study. In the design study, we wanted to observe the various sets of behaviors that arose when participants manipulated cubes of varying design characteristics. To measure these sets of behaviors, we designed several physical cubes (Figures 1a - f).

Each cube had a unique set of characteristics:

- $\quad$ Size: finger-sized cubes (Figure 1a), various small, medium and large cubes (Figure $1 \mathrm{~b}$ ).

- Texture: cube covered in various smooth (paper, lacquered) and rough (burlap, textured card) materials as well as squishable (Figure 1c) and organic, clay cubes (Figure 1f).

- Colour/Pattern: cubes with 2 colours placed in different arrangements on each face of the cube to create various patterns (Figure 1e).

- Weight: from heavy clay cubes (Figure 1f) to light paper cubes (Figure 1e).

- $\quad$ Shape: rhomboid (Figure 1f), star (Figure 1d).

- Sound: clay beads were added inside various cubes. As well, the squishy cubes caused a wheezing sound when being squeezed (Figure 1c). 


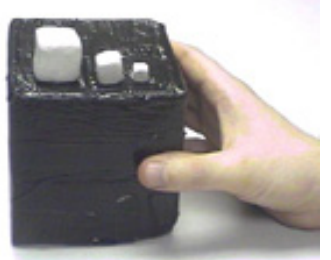

Figure 1a. Finger-sized cubes resting on a large cube.

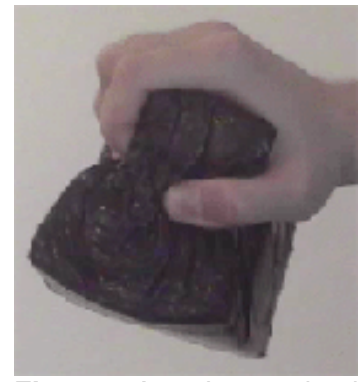

Figure 1c. Large-sized squishy cube.

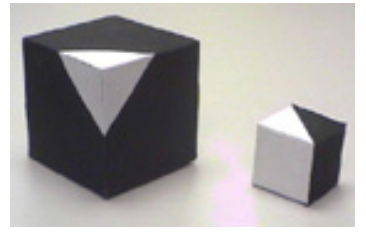

Figure 1e. Patterned cubes.

\subsection{Methodology}

Participants were given the various cubes and asked to answer 5 questions. The questions were designed to provoke volunteers to manipulate the cubes rather than deriving specific answers from participants:

1. If you had to pick one cube, which cube would you pick and why? Where would you keep it?

2. If you had to carry one of the cubes with you at all times, which one would it be? Why that one? How would you carry it?

3. One of the cubes is to be used as a control mechanism. Which cube should it be and show me how it works.

4. Create your own cube. Describe its form, functionality and the experience you would gain from using it. Describe in words, on paper or by drawing a picture what your cube would look like.

5. If one of the cubes was an alien life form, which one would it be? Describe how it lives.
From our questionnaires and observations, we expected to group cubes according to non-verbal dynamics, manipulations and user preference.

\subsection{Participants}

We solicited volunteers through a local new media centre via email and posted a call for participation on their website. Although the centre does not restrict their membership, we required that our study not include anyone under the age of 17 . All of our participants were involved in the arts, either as working artists or as art historians, and they came from a diverse social and ethnic background. Half our participants were women. Volunteers were informed prior to the test that they were participating in a design study that will assist in determining a framework for natural tangible interfaces.

\subsection{Measures}

To record our data, we use qualitative procedures, including observations and questionnaires. Observations were recorded with a video camera. Volunteers were asked to fill out a background questionnaire prior to the study. They were informed before beginning the test that they were going to be videotaped. Investigators recorded non-verbal manipulation. At the end of the design study, users completed a short post-questionnaire. Using multiple forms of observation and data collection allows for detailed evaluation and analysis of user behavior.

\subsection{Procedure}

Our design study took place over one week at a new media art gallery in northwest England (15). We divided our participants into two groups: one group completed the study individually and one collaboratively. Each participant interacted with two investigators. The assistant investigator was responsible for greeting and debriefing the volunteers and collecting questionnaires. A second investigator was responsible for videotaping. Both investigators were responsible for note taking during the study and for analyze data and questionnaires after the study.

Cubes were evaluated in four separate stages. First, participants filled out a pre-test questionnaire individually, which allowed us to gather background data about each participant. Next, we observed how participants interacted with each cube. On each testing day participants were given a list of 5 questions in which they conducted via the cubes. Investigators directly observed participants and collected data concerning these observed activities. As well as investigators directly observing participants, video cameras recorded (audio and visual) user activity. Thirdly, volunteers completed a post-test questionnaire individually and were asked to comment on the procedures, tasks, cubes and investigators. Lastly, and $\mathrm{HCl}$ expert and investigator analyzed the collected data. 


\subsection{Data Collection}

Individual responses were directly collected via the pre and post-test questionnaires and indirectly collected via video. When analyzing the video data, investigators first collected information on how each user individually handled the cubes (for example, selection patterns) and then how they collaboratively selected cubes. Secondly, investigators collected both individual and collaborative verbal responses to the 5 questions given at the beginning of the study. Occasionally participants queried investigators as to whether or not they had provided enough information to answer a particular question, and in all cases the investigators simply asked them to continue in whatever manner they wanted for as long as they felt necessary.

\section{RESULTS}

We grouped our results into four categories: observed manipulations helped us develop a classification of nonverbal dynamics; a classification of handling; user preferences; and, generalizations concerning grasp.

\subsection{Non-verbal Dynamics}

Non-verbal dynamics are the manipulations (gripping, not gesture) that take place when participants grip a cube. If the physical form of an interface fundamentally shapes the kinds of interactions that users can perform, what are the possible non-verbal dynamics that a user can perform with a cube? Our observations allowed us to classify the kinds of non-verbal dynamics a cube affords (Table 1). We classify these dynamics according to action (manipulation), description of the action, and events. The event is the type of action particular to that manipulation. For example, rotate is classified as a TURN event and is one that is dependent on the speed of turning, and possibly by the number of exposed sides.

TABLE 1 - Non-verbal dynamics of cubes.

\begin{tabular}{|c|c|c|}
\hline Action & Description & Events \\
\hline Rotate & $\begin{array}{l}\text { To turn cube about } \\
\text { an axis or centre in } \\
\text { a continuous, fluid } \\
\text { motion exposing } \\
\text { three sides of the } \\
\text { cube at one time. }\end{array}$ & $\begin{array}{l}\text { TURN + (exposed } \\
\text { sides })+(\text { speed })\end{array}$ \\
\hline Roll & $\begin{array}{l}\text { Impelling cube } \\
\text { forward by causing } \\
\text { it to turn over and } \\
\text { over on a surface. }\end{array}$ & $\begin{array}{l}\text { TURN + (surface } \\
\text { contact) } \\
\text { SPIN + (surface } \\
\text { contact) }\end{array}$ \\
\hline Twist & $\begin{array}{l}\text { To rotate cube } \\
\text { while taking a } \\
\text { curving path or } \\
\text { direction using the } \\
\text { wrist. }\end{array}$ & TURN + curve path \\
\hline Turn & $\begin{array}{l}\text { To cause cube to } \\
\text { move around an } \\
\text { axis or a centre, }\end{array}$ & $\begin{array}{l}\text { TURN (right, left, } \\
\text { up, down) }\end{array}$ \\
\hline
\end{tabular}

exposing one side at a time.

Throw To propel cube through the air by a forward motion of the hand and arm.

Flip

$$
\text { One fluid }
$$
movement to cause cube to turn over to expose the opposite side of the cube.

Spin To revolve the cube in a fast, fluid movement where all sides are exposed very quickly.

Hold To have or maintain cube in the grasp.

$\begin{array}{ll}\text { Shake } & \text { Sharp, fluid } \\ \text { movements up and }\end{array}$ down.

Shake

Sharp, fluid
movement side to
side.

Place To put cube in or as if in a particular place or position.

Squeeze Exert strong pressure on cube with hands or fingers.

Press Steady pushing or thrusting force exerted in contact with cube.

Pick up To take hold of and lift up.

Tap Strike cube quickly and lightly so that strike produces a slight sound.

Rub To move hand or fingers along the surface of the cube with pressure.

Fiddle To move the hands or fingers around the cube restlessly.

HOLD

movement)

(no

SHAKE

(up $\rightarrow$ down $\rightarrow$ up)

SHAKE

(down $\rightarrow$ up $\rightarrow$ down)

SHAKE

(left $\rightarrow$ right $\rightarrow$ left)

SHAKE

(right $\rightarrow$ left $\rightarrow$ right)

PLACE $\rightarrow$ HOLD

PLACE

PRESS + (force)

PRESS + (force) + (time)

PRESS $\rightarrow$ (up)

PRESS + (force) + (time) $+($ sound $)$

PRESS + (force) + (temperature) + (area)

Ambiguous movements

The actions Place and Pick up appear in the table since they begin and end most events and they include the grasping actions Hold and Press.

Programming the events describe in non-verbal dynamics will require that algorithms recognize the subtle differences between certain actions. This is an enormous task. For example, rotation consists of twofold axis (vertex), three-fold axis (edges) or four-fold axis (faces) as well as speed. To detect rotation, physical 
interfaces would need to determine both the axis type and the speed at which cube is being rotated. However, research has begun on possible implementation (16).

\subsection{Handling}

How a participant handles a cube determines the nonverbal dynamics that are available to them. We describe "handling" as managing with the hands by touching feeling and moving. All handling is impactive, in that action occurs only when hands come in contact with a cube.

Handling is divided into four categories:

- One-handed manipulation: using one hand to perform dynamics (Figure 2a).

- One-handed finger manipulation: using one hand and fingers to perform dynamics (Figure $2 b$ ).

- Two-handed manipulation: using two hands to perform dynamics (Figure 2c).

- Two-handed finger manipulation: using two hands and fingers to perform dynamics (Figure $2 \mathrm{~d}$ ).

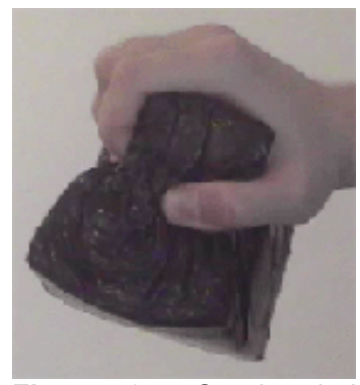

Figure 2a. One-handed manipulation.

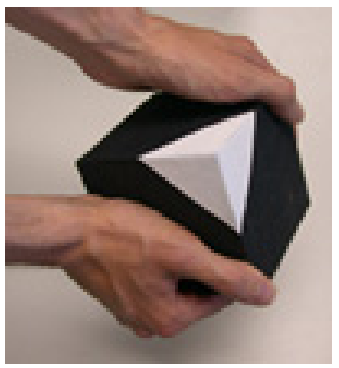

Figure 2c. Two-handed manipulation.

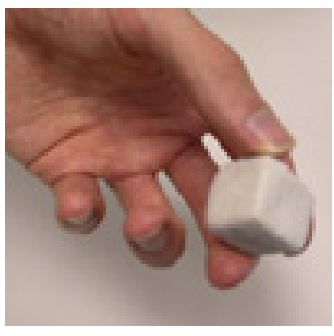

Figure 2b. One-handed finger manipulation.

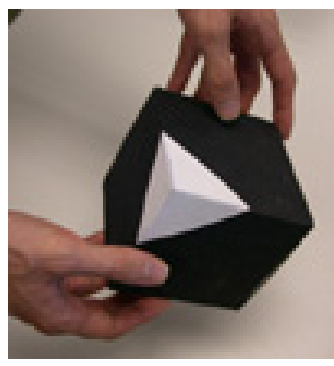

Figure 2d. Two-handed finger manipulation.
Each of the four handling categories can be applied to the dynamics described in Table 1 to develop handling conventions. Using ROTATE as an example, we can see how certain conventions would develop over time and continuous use:
- One hand rotation: Hold cube in palm of hand and rotate cube with fingers, turning cube away from you with thumb.

- One-handed finger rotation: Hold cube in between two fingers and rotate between fingers with thumb and free fingers.

- Two hand rotation: Hold cube with one hand and rotate it over the other; or, place one hand on the top of the cube and another on the bottom. Rotate cube with top hand and stabilize it with bottom hand.

- Two-handed finger rotation: Hold cubes by the corners and rotate.

\subsection{Preferences}

Every wearable user is physically different and will have a unique set of preferences. However, our results suggest that there are certain attributes that are general to all physical interfaces and that may make one physical interface more desirable than another.

Form over function. Participants were drawn to cubes that broke from the usual form of a cube, such as a rhomboid and one with an extruded edge. As well, participants were able to explore more freely cubes that didn't already contain some conventional meaning or function. For example, participants rejected cubes that simply looked like gift boxes.

Feedback prolongs interaction. Some of the cubes "reacted" to user interaction. For example, cubes with beads in them produced rolling sounds and soft cubes retained a deformed shape after being squeezed. Participants interacted with these cubes more often than the others and would hold them for long periods of time.

Wider multi-sensory experience prolongs interaction. Visual quality is not enough to sustain attention. Cubes that offered two or more types of sensory experience were favoured. Clay cubes felt organic and left a residue on hands would appeal to users' sense of touch and smell. Multi-sensory experiences blend visual and tactile texture, colour, smell, sound, size, form, and weight.

Some degree of weight is desirable. Having some degree of weight is desirable. Weight allows people greater control over manipulation. Interfaces must be heavy enough that users are aware of the object but light enough that it can be carried or held for long periods of time. Weight should be adjusted accordingly.

Size is relative to the user and application. In terms of handling, the bigger something is the harder it is to carry but easier to find. Conversely, the smaller something is the easier it is to carry and the harder it is to find. As with weight, the interface needs to be designed so that it does not impinge too much on users' space. Participants suggested that smaller cubes could be attached to a key fob or worn as jewellery. Larger cubes could be useful for low-mobility users. If the application required that the 
user carry the cube in their pocket, then smaller cubes were favoured.

\subsection{Grasp}

Our study alerted us to how certain physical factors of cubes affect participants' ability to grasp cubes, and therefore would affect their ability to manipulate cubes. Our findings are consistent with studies in prehensor grip (17).

Geometry affects grip. Geometry influenced the amount of grip force needed for participants to securely hold the object. Participants prefer cubes that fit naturally in the hand. For example, the perfect cube did not fit well in participants' hands and thus was difficult for onehanded manipulation. Cubes that had some curviness were easier and more comfortable to grip. For example, the rhombus fit comfortably in the hand and the star fit well between the fingers.

Surface area enhances grip. The amount of surface contact area of a cube enhances security of grip.

Flexibility of materials aids in grip. Flexible material lessens the amount of force users need to grip a cube. When a cube is held in the hand, the hand deforms to conform to the cube's shape. If a cube is flexible, then the amount of force needed to hold the cube decreases. For example, participants were able to more easily manipulate cubes that were wrapped in bubble sheet or squishy because of their flexibility. This flexibility allows users to pick up cubes much more easily.

\section{DESIGNING PYSICAL INTERFACES FOR WEARABLE COMPUTING}

An understanding of affordance is critical to the success of user-centric physical interfaces for wearable computing. Our study determined that physical interfaces should share the same functionality but have adjustable and modular physical attributes. Functionality for wearable computing, then, should be established by a set of well-defined, non-verbal dynamics.

Because not all users have the same motor control, wearable physical interfaces should be adjustable to fit user preference and ability. For example, in some cases, users with lower-motor skills will require larger physical interfaces with higher flexibility and conversely users with high motor skills will require smaller interfaces with lower flexibility.

Physical attributes of wearable physical interfaces must be adjusted to fit the type of mobile activity. Users may be engaged in everyday activity such as shopping, driving and running or they may be completing a wearable-specific task such as mobile maintenance or underwater photography. Interfaces should be modular to adjust to their environment and activity.

Non-verbal dynamics combined with the type of mobile activity will determine whether a one-handed or twohanded physical interface is necessary.
Wearable users may be carrying keys, money, tools or other portable objects. Designers must consider that a physical interface may not be the only object users are carrying and adjust its physical attributes based on this knowledge.

Currently, we continue to perform design studies with a prototype wearable physical interfaced called Cubicle (16). We anticipate that such studies will reveal general usability flaws in the device design, which will have to be corrected through redesign. For example, type of events and dynamics may need adjustment according to user feedback. Such practice will help us to produce desirable and appropriate device features that are user-centric.

Since the Cubicle is a unique physical interface that is being targeted to a general population of users, we expect that it has implications for use with wider applications within the mobile and wearable computing community. It is expected that the results of future studies will provide some specific benchmarks that can be used in the development of similar systems.

\section{FUTURE CONSIDERATIONS}

Our intention of this study was to lay the foundations for classifying non-verbal dynamics for physical interfaces and as a result is exploratory and qualitative. However, our study revealed a number of observations that provide the groundwork for future research in non-verbal dynamics.

Design studies are an economical, quick and easy way to gain an enormous amount of qualitative information about interaction with tools. In just a few weeks, we were able to design, implement and analyse prototype interfaces.

In our observations, we noticed that on a number of occasions participants would group small cubes together or place them on top of larger cubes. This behaviour is consistent with Kirsh's findings that humans use spatial organization to reduce cognitive load (18). We expect that this kind of behaviour will increase with use of multiple cubes.

We would like to further explore what Fels' describes as intimacy and embodiment of objects (6). We expect that long-term usage of cubes will bring out people's inherent tendency towards animism and creating relationships with objects.

\section{CONCLUSIONS}

Our design study helped us to determine the natural affordances of cubes. From this study we were able to develop a classification of non-verbal dynamics, define and provide categories of handling, identify general design preferences, and to propose conventions that may develop from continuous cube use. This work provides an important first step in the design of physical interfaces for wearable and mobile computing applications. 


\section{ACKNOWLEDGEMENTS}

The presented research is supported by the UK Engineering and Physical Science Research Council (EPSRC) under grant GR/S08848/01 ("Multi-Sensor Perceptive Interfaces in Wearable and Ubiquitous Computing") and as part of the Equator IRC (GR/N15986/01 - "Technological Innovation in Physical and Digital Life"). We would like to thank folly and the artists who volunteered for our study.

\section{REFERENCES}

1. Huang, P. 2000. Promoting wearable computing: A survey and future agenda. Technical Report TIKNr.95, Computer Engineering and Networks Laboratory, Swiss Federal Institute of Technology.

2. Rekimoto, J., Ullmer, B., and Oba, H. 2000. ToolStone: Effective use of the physical manipulation vocabularies of input devices. In Proc. of UIST.

3. Rekimoto, J. 1996. Tilting operations for small screen interfaces. In Proc. of UIST.

4. Bartlett, J. F. 2000. Rock'n'Scroll is here to stay. IEEE Computer Graphics and Applications, 20(3):40-45.

5. Benford S., Schnadelback H., Koleva B., Paxton M., Anastasi R., Greenhalgh C., Gaver B. 2002. Sense and Sensability: a framework for designing physical interfaces. Technical Report Equator-02009, Equator, September.

6. Fels, Sidney S. 2000. Intimacy and Embodiment: Implications for Art and Technology. In Proc. of the ACM Conference on Multimedia, 13-16.

7. Baudel, T. \& Beaudouin-Lafon, M. 1993. Charade: Remote Control of Objects using FreeHand Gestures. Communications of the ACM, 36(7): 2835.

8. Myers, B. A. 1995. User Interface Software Tools. ACM Trans. Computer-Human Interaction, 2(1): 64-103.

9. Fitzmaurice G., Ishii H., Buxton W. 1995. Bricks: Laying the Foundations for Graspable User Interfaces. ACM Proceedings CHI'95, Denver, Colorado.

10. Buttolo, P. 1996. Characterization of Human Pen Grasp With Haptic Displays. PhD Dissertation. University of Washington.

11. Singleton, W.T. 1982. The Body at work: biological ergonomics. Cambridge, University Press.

12. Pheasant, S. 1992. Bodyspace: anthropometry, ergonomics and design. Taylor \& Francis.
13. Norman, D. A. 1988. The Design of Everyday Things. New York: Doubleday.

14. Dix, A., Finlay, J., Abowd, G., Beale, R. 1998. Human-Computer Interaction (2nd ed.). Essex: Prentice Hall.

15. folly media arts organisation. http://www.folly.co.uk.

16. Van Laerhoven, K., Villar, N., Schmidt, A., Kortuem, G., Gellersen, H. 2003. Using an Autonomous Cube for Basic Navigation and Input. International Conference on Multimodal Interfaces, Vancouver, Canada.

17. Shaperman, J., LeBlanc, M. 1995. Prehensor Grip for Children: A Survey of the Literature. Journal of Prosthetics and Orthotics, 7(2) pp. 61-64.

18. Kirsh D. 1995. Complementary Strategies: Why we use our hands when we think. In Proc. of the Seventeenth Annual Conference of the Cognitive Science Society. Hillsdale, NJ: Lawrence Erlbaum. 